\title{
Editorial: advanced catalytic materials for environmental application
}

\author{
Junhua Li
}

Published online: 15 August 2014

(c) Science China Press and Springer-Verlag Berlin Heidelberg 2014

Environmental protection is directly related to the sustainable development of the economy and society. Controlling the release of major air and water pollutants remains a daunting challenge in China. The removal of major air pollutants $\left[\mathrm{PM}, \mathrm{SO}_{2}, \mathrm{NO}_{x}\right.$, volatile organic compounds (VOCs), and $\mathrm{Hg}$ ] from stationary and mobile sources is becoming increasingly urgent to improve air quality. Additionally, the removal of numerous industrial and natural chemical compounds from contaminated water systems is essential to maintaining the availability of clean water. Therefore, cost-effective and appropriate air pollution control and water treatment technologies must be explored and implemented. Regarding air pollution control, the selective catalytic reduction of $\mathrm{NO}_{x}(\mathrm{SCR})$ by ammonia and hydrocarbons over metal oxide and zeolite catalysts is discussed; non-toxic transition metal oxides supported on titania and zeolite matrices are emerging new catalysts. The removal of $\mathrm{Hg}$ from flue gas using catalysis is also a new research direction in this field. The oxidation of VOCs using photo- and thermal catalysis for indoor air quality improvement is reviewed based on recent research progress achieved worldwide. Regarding wastewater treatment, the catalytic elimination of inorganic and organic pollutants from wastewater is presented. Additionally, mechanisms for the abatement of air and water pollutants using catalysis are discussed in detail. Finally, several aspects should be aimed in future research of environmental catalysis for the abatement of major pollutants in air and water. For instance, the development of environment-friendly catalysts that have attracted much attention in both the academic and industrial fields should be enhanced. Significant efforts should be made towards the development of highly active and durable catalysts, and anti-fouling catalysts for long-term successful treatments. For the selectivity of pollutants transformation to harmfulness should be more concerned by adjusting heterogeneous catalytic processes. Further efforts should be made to gradually improve material or reactor design for pilot and demonstration purposes. Moreover, much work is required to combine catalytic processes with other remediation technologies.

SPECIAL TOPIC: Advanced Catalytic Materials for Environmental Application

J. Li (ه)

State Key Joint Laboratory of Environment Simulation and Pollution Control, School of Environment, Tsinghua University, Beijing 100084, China

e-mail: lijunhua@tsinghua.edu.cn 